\title{
Nuclear Magnetic Resonance-Based Current-Voltage Source
}

Cheol Gi Kim, E. R. Williams, Member, IEEE, H. Sasaki, S. Ye, P. T. Olsen, and W. L. Tew, Member, IEEE

\begin{abstract}
A one-ampere current has been stabilized using nuclear magnetic resonance techniques. A pair of tandem solenoids produces two uniform magnetic fields in opposite directions, and these fields are not affected by external magnetic shielding. The current and background field are controlled to within $0.1 \mathrm{ppm}$ over an $8 \mathrm{~h}$ period.
\end{abstract}

\section{INTRODUCTION}

$\mathrm{I}$ $\mathrm{N}$ this paper, we introduce a new solenoid geometry for producing magnetic fields. This geometry reduces the problem of background noise and drifts due to thermal noise. It also allows the use of magnetic materials that were not allowed or caused problems in earlier nuclear magnetic resonance (NMR)-based current sources. In our laboratory, we need current and voltage sources that are stable at about the $0.01 \mathrm{ppm}$ level. A Josephson array has sufficient accuracy to meet our need, but it is difficult to connect it directly to an experiment that is difficult to shield against RF interference and it is difficult to operate unattended for $24 \mathrm{~h}$ a day. Ultimately, it would be useful if this standard could be transported after calibration. We report much progress toward building this intermediate standard, and show that our new NMR scheme has produced a current-voltage source that is comparable to the best Zener diode references. This present current source already has less drift than commonly used current sources based on mercury battery references. The technique is unique and the promise is sufficient to warrant publication before the final studies and possible further improvements are complete.

Locking a magnetic field to a nuclear magnetic resonance (NMR) frequency is a well-established technique

\footnotetext{
Manuscript received June 12, 1992; revised September 21, 1992, This work was supported by the Calibration Coordination Group of the U.S Department of Defense.

C. G. Kim was on leave at the National Institute of Standards and Technology, Gaithersburg, MD 20899. He is with the Magnetics Laboratory, Korea Research Institute of Standards and Science, Magnetics Laboratory, Taedok Science Town, Taejon, Korea.

E. R. Williams, S. Ye, and P. T. Olsen are with the Electronics and Electrical Engineering Laboratory, Electricity Division, National Institute of Standards and Technology, U.S. Department of Commerce, Technology Administration, Gaithersburg, MD 20899.

H. Sasaki was on leave at the National Institute of Standards and Technology, Gaithersburg, MD 20899. He is with the Electrotechnical Laboratory, Tsukuba, Japan.

W. E. Tew was with the National Institute of Standards and Technology, Gaithersburg, MD 20899. He is now with the Process Measurements Division, National Institute of Standards and Technology, Gaithersburg, MD 20899 .

IEEE Log Number 9206505.
}

in high-resolution NMR experiments [1]. Fluctuations in the magnetic field are detected by NMR and compensated by a servocurrent. Using this method, a magnetic field has been stabilized to better than 1 in $10^{9}$ [2]. At the Electrotechnical Laboratory (ETL) in Japan, a current from an electromagnet under NMR control has been stabilized to $0.2 \mathrm{ppm} / \mathrm{h}$ [3]. The limitation to current stability at ETL was the change in dimensions of the electromagnet caused by heating in the windings. The magnetic hysteresis of the ferrous material prevented return to the same current each time the magnet was energized. In the present experiment, these limitations of the electromagnet current source have been reduced by using low thermal expansion fused silica as the solenoid former and a coil geometry that eliminates ferromagnetic material in the magnetic circuit. At the Mendeleev Institute of Metrology (VNIIM) in St. Petersburg [4], a dual-field current system using fused silica former was built that clearly demonstrated the usefulness of NMR techniques for making a precision current source. They made currents stable to at least 0.05 $\mathrm{ppm} / \mathrm{h}$. Our present system differs from that built in VNIIM mainly in the fact that our geometry allows us to add magnetic shielding around the experiment. Our present geometry combines many of the advantages of the VNIIM and ETL approaches.

\section{EXPERIMENT}

A set of two solenoids is used to produce two uniform magnetic fields in opposite directions, and both fields are linear with the current. Two water samples in the center of each solenoid can be used to measure two NMR frequencies that are proportional to the field at each location. A constant current and a constant background field are produced by employing two servosystems that keep both NMR frequencies constant. Thus, at the same time a current source is made, a sensitive vector magnetometer is made.

\section{Tandem Fields}

Fig. 1 shows the coil configuration used to produce two uniform magnetic fields pointing in opposite directions. Two solenoids wound on a fused silica form are wired so that the current in each has an opposite sense. The gradient from one solenoid greatly limits the uniformity at the center of the other. A second fused silica form has two additional solenoids (outer windings of the upper and 


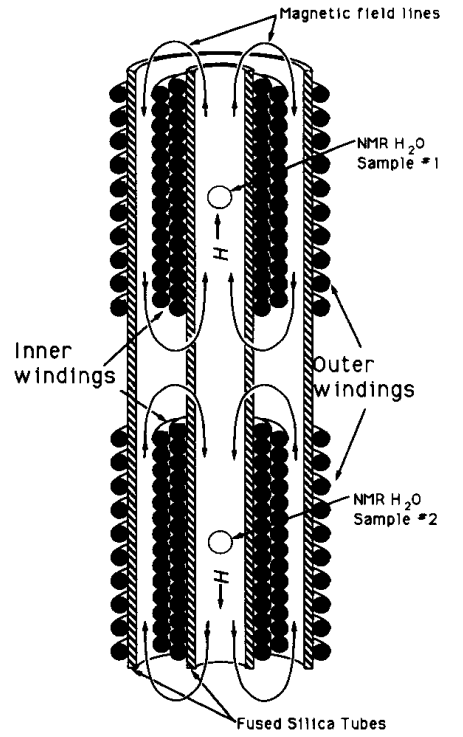

Fig. 1. Tandem fields produced by coaxial coils.

lower sets), and the dimensions and turns ratio have been chosen so that the number of area-turns of the outer coils is the same as the number of area-turns of the inner coils. Therefore, each set of inner and outer coils has no dipole field [5]. In fact, there is almost no field outside the pair, so the gradient produced at the neighboring set's center is much less, and the interaction with the ferromagnetic shields that surround the coils is small (see Fig. 2). A small price is paid for containing the return flux. The magnitude of the field produced at the center of each coil set is reduced by about the square of the ratio of the inner to outer diameters. The two outer coils are axially offset slightly from the inner coils to cancel the small remaining gradient produced by the other coil set. Additional subcurrents are added to sections of the inside coils to compensate for higher order gradients [6], but these small subcurrents only have a small contribution to the total field at the centers. Fig. 3 shows the details of the wiring. The inner coils have two layers of windings, one on top of the other and having the same helical sense. The outer coils are machined to have two helical coils (sometimes called bifilar). The pitch diameters and number of turns for each coil or subcoil are given in Table I. The negative percentages indicate that the field produced by that coil is in the opposite direction from the total field at the center. Notice that the total adds to $100 \%$. The fields at the center of each set are uniform to $20 \mathrm{ppm}$ over a $3 \mathrm{~cm}$ diameter.

\section{NMR Measurements}

The frequencies of both fields are "locked" to the same frequency, $62025 \mathrm{~Hz}$, using flowing water NMR. Fig. 4 shows the arrangement used to provide a continuous $\mathrm{dc}$ signal from the flowing water NMR. The water is polarized in a $0.3 \mathrm{~T}$ field outside the magnetic shield, and then

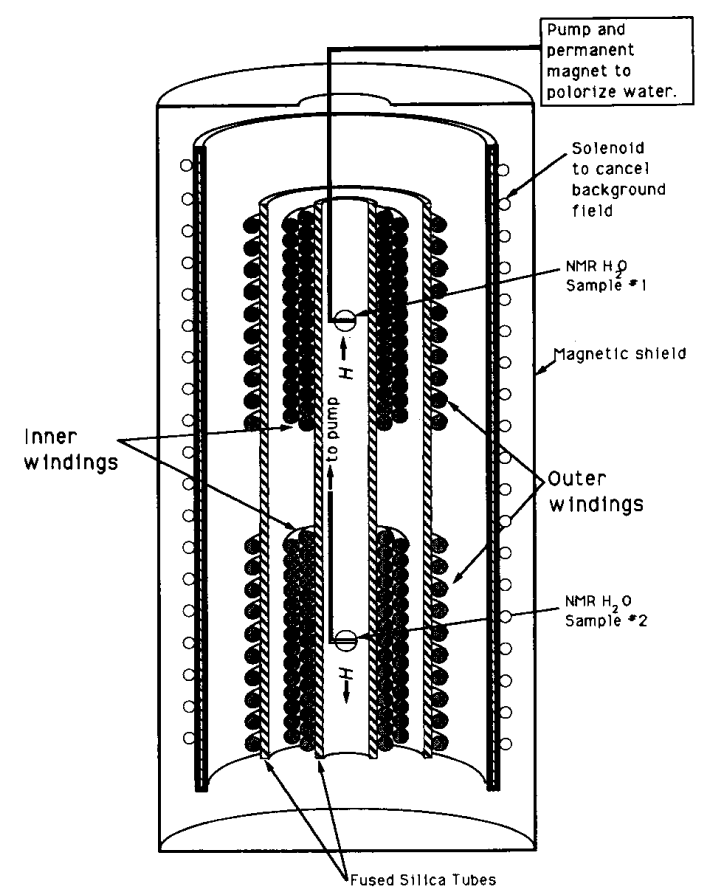

Fig. 2. Schematic drawing of overall system.

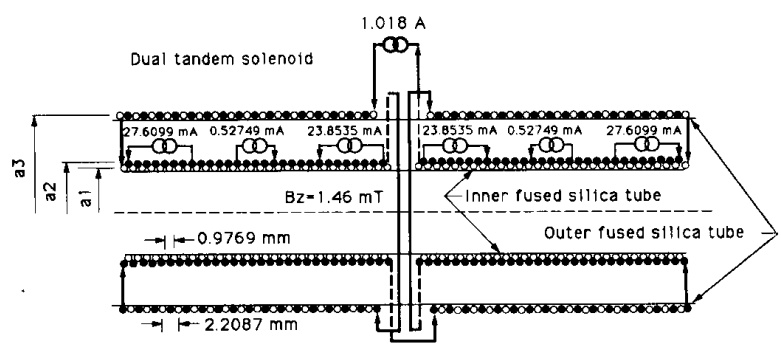

Fig. 3. Wiring arrangement for the double set of coils.

pumped into a $2.5 \mathrm{~cm}$ diameter sphere at the center of each solenoid. Just before entering the sphere, a small coil applies a constant amplitude RF field perpendicular to the dc field. The magnitude of this field is adjusted so that each proton passing through sees a $\pi / 2$ pulse. (A $\pi / 2$ pulse means that each proton sees an RF field of the proper magnitude and duration such that all of the protons will have a precession frequency in phase with each other.) A detector coil surrounding the sphere is perpendicular to both the $\pi / 2$ field and the dc magnetic field. Each sample has a lock-in detector whose dispersion signal is fed back to control either the main current or the current that controls the background field. The linewidth of the NMR signal is limited by the magnetic field gradient and is $1.0 \mathrm{~Hz}$ for the main current signal and $2.4 \mathrm{~Hz}$ for the background field signal compared to about $0.1 \mathrm{~Hz}$ if the fields were uniform. This line broadening is a major limitation to the present accuracy. 
TABLE I

Parameters for One Set of Colls. The Tandem Set has All Currents Reversed

\begin{tabular}{llcrr}
\hline \multicolumn{1}{c}{ Layer Name } & $\begin{array}{c}\text { Radius } \\
(\mathrm{cm})\end{array}$ & $\begin{array}{c}\text { Number } \\
\text { of Turns }\end{array}$ & $\begin{array}{c}\text { Current } \\
(\mathrm{A})\end{array}$ & $\begin{array}{c}\text { Percentage of } \\
\text { Total Field }\end{array}$ \\
\hline First Inner Layer & $a 1=3.675$ & 290 & $I_{\text {main }}=1.018$ & 89.5 \\
Second Inner Layer & $a 2=3.746$ & 290 & $I_{\text {main }}$ & 89.5 \\
First Outer Layer & $a 3=5.596$ & 128 & $-I_{\text {main }} /$ turn)
\end{tabular}

${ }^{a}$ Approximate values, adjusted to maximize NMR signal.

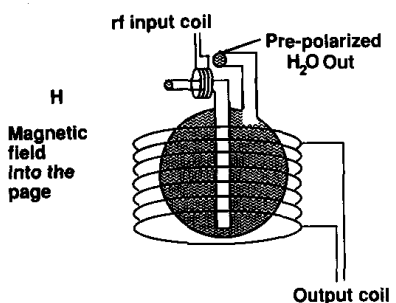

Fig. 4. Flowing water NMR schematic. The RF coil provides the $p / 2$ pulse, and the output coil detects the resonance frequency.

\section{Apparatus Assembly}

The sets of coils wound on fused silica forms are secured together with low thermal expansion plastic end caps. Copper tubing is wound on the outside of each coil, and cooling water flowing through these tubes is used to control the temperature of the solenoids. Around these fused silica coils is a single-layer solenoid wound on a PVC tube. (See Fig. 2.) A small current in this solenoid is used to control the background field at both NMR samples. Two Mu-metal shields surround the entire apparatus. The shields must shield out the component of the background field perpendicular to the axis of the solenoids. The polarizing magnet is outside the shields about $30 \mathrm{~cm}$ from the shields. Our servoscheme only cancels the axial component of the fields. The shields reduce the perpendicular component to less than $0.1 \mu \mathrm{T}$, which produces less than $0.01 \mathrm{ppm}$ correction because it adds in quadrature.

\section{Voltage Measurement}

The $1 \mathrm{~A}$ current is passed through a $1 \Omega$ resistor that has very low drift and a small power coefficient. A nanovoltmeter is used to compare the voltage with a $\mathrm{Ze}$ ner reference voltage standard.

\section{The Results}

\section{Short-Term Noise}

Fig. 5 shows the short-term stability of the system. A $3 \mathrm{~h}$ trace of the NMR source versus a Zener reference is plotted with a calibration made by increasing or decreasing the frequency by $0.1 \mathrm{~Hz}$. The lower trace is obtained when the NMR source is replaced by another Zener ref-

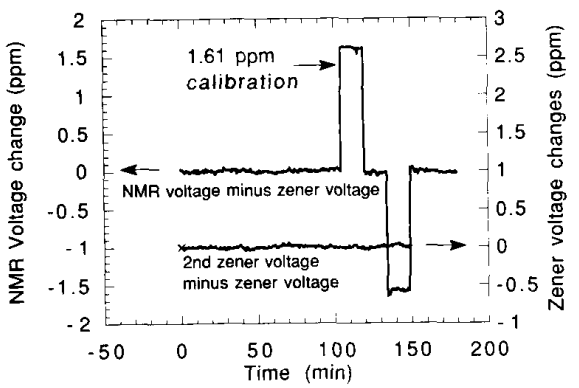

Fig. 5. Short-term noise. NMR versus Zener reference, scale to the left and Zener reference versus Zener reference, scale to the right. There is a $1 \mathrm{ppm}$ offset between the scales.

erence. Presently, the noise of the current source is similar to that of the Zener reference. With an improved linewidth in the NMR signal, this noise will be reduced further.

\section{On-Off Noise}

Fig. 6 demonstrates how well the system works when turned off, and then on again 1 min later. The current and magnetic field were turned off four different times, as indicated by the arrows. These data show that there is no problem at the $0.05 \mathrm{ppm}$ level with magnetic hysteresis from the shields or other problems in locking the servosystem. Longer term on-off cycles must still be demonstrated, but improved temperature control of the solenoids should be completed first.

\section{Hourly Noise}

Fig. 7 is our best record to date of the hourly drift rate. The bandwidth of the measurement system is determined by the $60 \mathrm{~s}$ integration time used for the data shown in Figs. 5 and 7 . Note that the temperature of the solenoid still is a major source of drift. The temperature coefficient is not too big, about $1 \mathrm{ppm} / \mathrm{K}$, but the time rate of change of the temperature is an unexplained source of noise. Better temperature control should improve the results.

\section{Summary}

A current/voltage source is operated at a level better than $0.1 \mathrm{ppm}$, and shows promise of outperforming Zener 


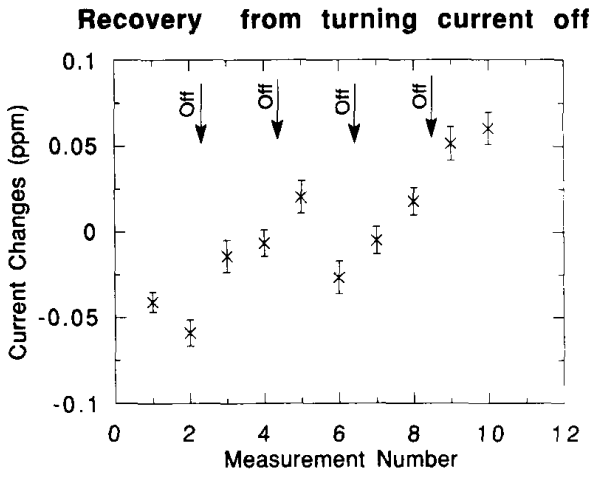

Fig. 6. Recovery from turning off current and magnetic field.

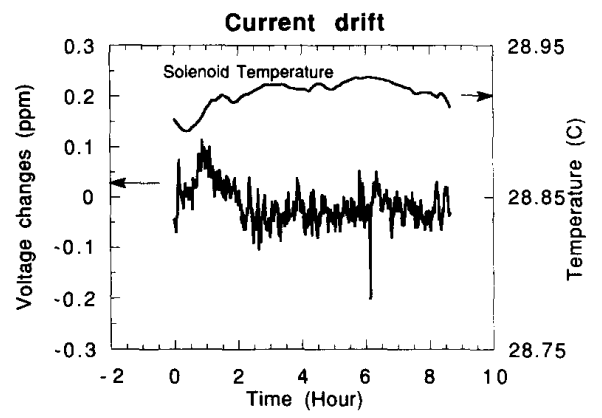

Fig. 7. Voltage changes over $9 \mathrm{~h}$ period.

diodes in applications requiring rugged voltage references. Such a standard will still need to be calibrated against a Josephson array, but if some improvements are made, the time between calibrations can be long. The ability to recover from being turned off shows its potential as a transport standard. Improvements in the NMR are required to realize this goal fully, but techniques such as Ramsey oscillatory field techniques [7] should allow this improvement. The result of the VNIIM group [4] shows that an NMR-based system can do better than reported here. Assuming that we can reproduce their NMR techniques, we should have a shielded transportable system that is significantly more stable than Zener diode references.

\section{REFERENCES}

[1] R. C. LaForce, G. LaForce, and G. R. Hansen, "A proton magnetic field controller," Rev. Sci. Instrum., vol. 43, pp. 1695-1698, Nov. 1972.

[2] S. Kan, P. Gonord, M. Fan, and M. Sauzade, "Automatic NMR fieldfrequency lock-pulsed phase locked loop approach," Rev. Sci. Instrum., vol. 49, pp. 785-789, June 1978 .

[3] H. Sasaki, A. Miyajima, N. Kasai, and H. Nakamura, "High-stability DC-current source using NMR locked technique," IEEE Trans. Instrum. Meas., vol. IM-35, pp. 642-643, Dec. 1986.

[4] E. V. Blinov, P. P. Kuleshov, V. N. Khorev, A. E. Shilov, and V. Ja. Shifrin, "Ampere realization via proton gyromagnetic ratio," Document CCE/80-30 submitted to the 18th Meeting of the CCE, 1988.

[5] E. R. Williams and P. T. Olsen, "A method to measure magnetic fields accurately using Ampere's law," IEEE Trans. Instrum. Meas., vol. IM-27, pp. 467-469, Dec. 1978.

[6] E. R. Williams et al., "A low field determination of the proton gyromagnetic ratio in water," IEEE Trans. Instrum. Meas., vol. 38, pp. 233-237, Apr. 1989.

[7] G. L. Greene et al., "Measurement of the neutron magnetic moment," Phys. Rev. D, vol. 20, pp. 2139-2153, Nov. 1979. 